\title{
Fermi Surface of the Most Dilute Superconductor
}

\author{
Xiao Lin, ${ }^{1,2}$ Zengwei Zhu, ${ }^{1}$ Benoît Fauqué, ${ }^{1}$ and Kamran Behnia ${ }^{1}$ \\ ${ }^{1}$ LPEM (UPMC-CNRS), Ecole Supérieure de Physique et de Chimie Industrielles, 75005 Paris, France \\ ${ }^{2}$ Department of Physics, Zhejiang University, Hangzhou, 310027, China
}

(Received 5 December 2012; published 15 April 2013)

\begin{abstract}
The origin of superconductivity in bulk $\mathrm{SrTiO}_{3}$ is a mystery since the nonmonotonous variation of the critical transition with carrier concentration defies the expectations of the crudest version of the BCS theory. Here, employing the Nernst effect, an extremely sensitive probe of tiny bulk Fermi surfaces, we show that, down to concentrations as low as $5.5 \times 10^{17} \mathrm{~cm}^{-3}$, the system has both a sharp Fermi surface and a superconducting ground state. The most dilute superconductor currently known therefore has a metallic normal state with a Fermi energy as little as $1.1 \mathrm{meV}$ on top of a band gap as large as $3 \mathrm{eV}$. The occurrence of a superconducting instability in an extremely small, single-component, and barely anisotropic Fermi surface implies strong constraints for the identification of the pairing mechanism.
\end{abstract}

DOI: 10.1103/PhysRevX.3.021002

\section{INTRODUCTION}

$\mathrm{SrTiO}_{3}$ is a large-gap transparent insulator, which, upon the introduction of $n$-type carriers, undergoes a superconducting transition below $1 \mathrm{~K}$. Discovered in 1964 [1], it is the first member of a loose family of "semiconducting superconductors" [2], which now includes column-IV elements [3]. During the last decade, attention has been focused on the interface between $\mathrm{SrTiO}_{3}$ and other insulators [4] or a vacuum [5], which is a two-dimensional metal with a superconducting ground state $[6,7]$.

According to Mott, in a doped semiconductor, when the average distance between the dopants $\left(d=n^{-1 / 3}\right)$ becomes a sizeable fraction of the effective Bohr radius $a_{B}^{*}$, a metal-insulator transition occurs. Quantitatively, this Mott criterion for the critical concentration is expressed as $n_{c}^{1 / 3} a_{B}^{*}=0.26$ and has been observed to hold in a wide range of semiconductors [8].

Insulating $\mathrm{SrTiO}_{3}$ is dubbed a quantum paraelectric. At low temperature, its static dielectric constant becomes 4 orders of magnitude larger than a vacuum [9]. Since the effective Bohr radius is proportional to the dielectric constant, this large dielectric constant implies a very long Bohr radius. Therefore, following the Mott criterion, the critical density for a metal-insulator transition is expected to be much lower than in ordinary semiconductors, and, indeed, doped $\mathrm{SrTiO}_{3}$ displays a finite zero-temperature conductivity down to carrier concentrations as low as $n=8 \times 10^{15} \mathrm{~cm}^{-3}$ [10], orders of magnitude lower than the threshold of metallicity in silicon $\left(3.5 \times 10^{18} \mathrm{~cm}^{-3}\right)$ or in germanium $\left(3.5 \times 10^{17} \mathrm{~cm}^{-3}\right)[8]$.

Published by the American Physical Society under the terms of the Creative Commons Attribution 3.0 License. Further distribution of this work must maintain attribution to the author(s) and the published article's title, journal citation, and DOI.
At still higher doping levels, the system becomes a superconductor when it is $n$ doped by one of the three possible routes: substituting titanium with niobium [11-13], strontium with lanthanum [14], or removing oxygen [11]. Intriguingly, the superconducting ground state is restricted to a limited doping window [11-14]. Thanks to the remarkably high mobility of electrons, another consequence of the large dielectric constant [10], quantum oscillations are observable and have been reported both in the bulk $[15,16]$ and in the twodimensional [17-20] samples.

The main subject of the present work is to yield a quantitative description of the emerging Fermi surface and to clarify its relationship with the superconducting ground state. Our work addresses two hitherto unanswered questions. (i) Is there a threshold in carrier concentration for the emergence of superconductivity? (ii) Does the normal state of such a low-density superconductor have a well-defined Fermi surface, or is it an impurity-band metal? We provide answers to these questions by a study of the low-temperature Nernst effect (a very sensitive probe of tiny Fermi surfaces [21-23]) in both oxygenreduced and $\mathrm{Nb}$-doped $\mathrm{SrTiO}_{3}$ across a wide (i.e., 3-orders-of-magnitude) window of carrier density.

We find that superconductivity persists down to a carrier concentration that is significantly lower than what was previously believed. This result firmly establishes $n$-doped $\mathrm{SrTiO}_{3}$ as the most dilute known superconductor with a carrier density as low as $5.5 \times 10^{17} \mathrm{~cm}^{-3}$, which corresponds to the removal of one oxygen atom out of $10^{5}$. At this carrier concentration, giant Nernst quantum oscillations with a single frequency are observed. The superconductivity persists even in the presence of a single, barely filled, and almost isotropic band. We will argue that this context is radically different from what one finds in conventional phonon-mediated BCS superconductors. Thus, more than four decades after its discovery, $\mathrm{SrTiO}_{3}$ 
emerges from this study as a candidate for unconventional superconductivity.

\section{EXPERIMENTAL}

The study was carried out on bulk commercial $\mathrm{SrTiO}_{3}$ single crystals. Oxygen-deficient samples were obtained by annealing nominally stochiometric samples. The Hall and longitudinal resistivity of the samples were measured, and the carrier dependences of mobility and
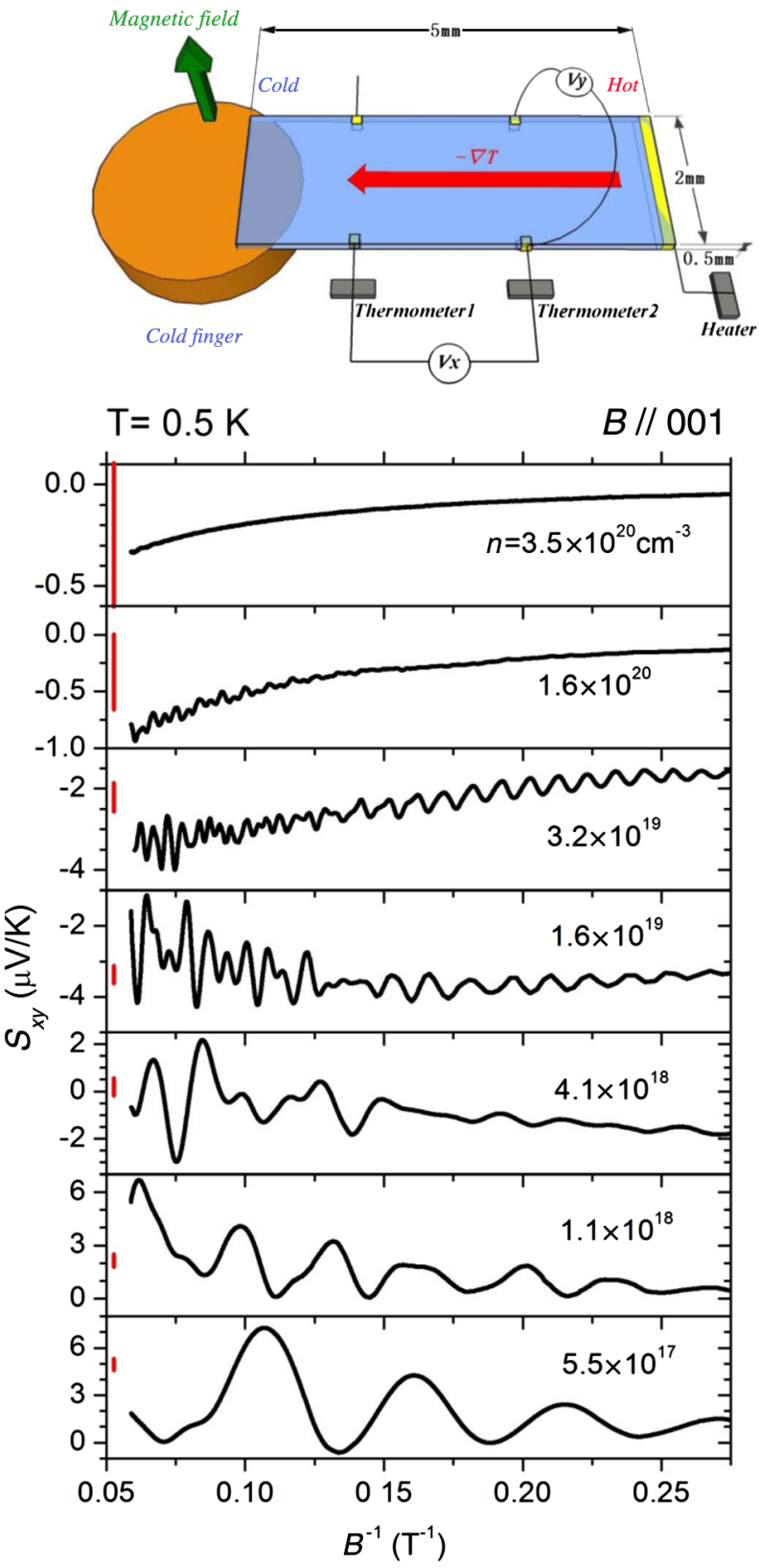

FIG. 1. Top: The experimental setup for measuring Nernst and Seebeck coefficients of bulk chemically doped $\mathrm{SrTiO}_{3}$. Bottom: As the carrier density is reduced in $\mathrm{SrTiO}_{3}$, the Nernst signal shows oscillations with larger amplitude and longer periodicity. In each panel, the vertical red bar represents a constant scale of $0.7 \mu \mathrm{V} / \mathrm{K}$. room-temperature resistivity were found to be compatible with early reports by Fredrikse and co-workers [24,25] and recent work by Spinelli and co-workers [10]. In order to obtain Ohmic contacts, gold was evaporated on the samples and heated up to $550^{\circ} \mathrm{C}$. The typical contact resistance was a few Ohms at room temperature and well below $1 \mathrm{Ohm}$ at low temperature. A one-heater-twothermometers setup was used to measure all transverse and longitudinal electric and thermoelectric coefficients (resistivity, Hall, Seebeck, and Nernst). A sketch of the setup is shown in the upper panel of Fig. 1. At low temperatures, a noise level of $1 \mathrm{nV}$ was achieved. The accuracy of the thermal gradient across the sample was checked by retrieving the Wiedemann-Franz relation between thermal and electric conductance in each sample. Details on the sample preparation and characterization are given in the Supplemental Material [26].

\section{RESULTS AND DISCUSSION}

Figure 1 presents the variation of the Nernst signal $\left(S_{x y}=E_{y} /-\nabla_{x} T\right)$ as a function of the inverse of the

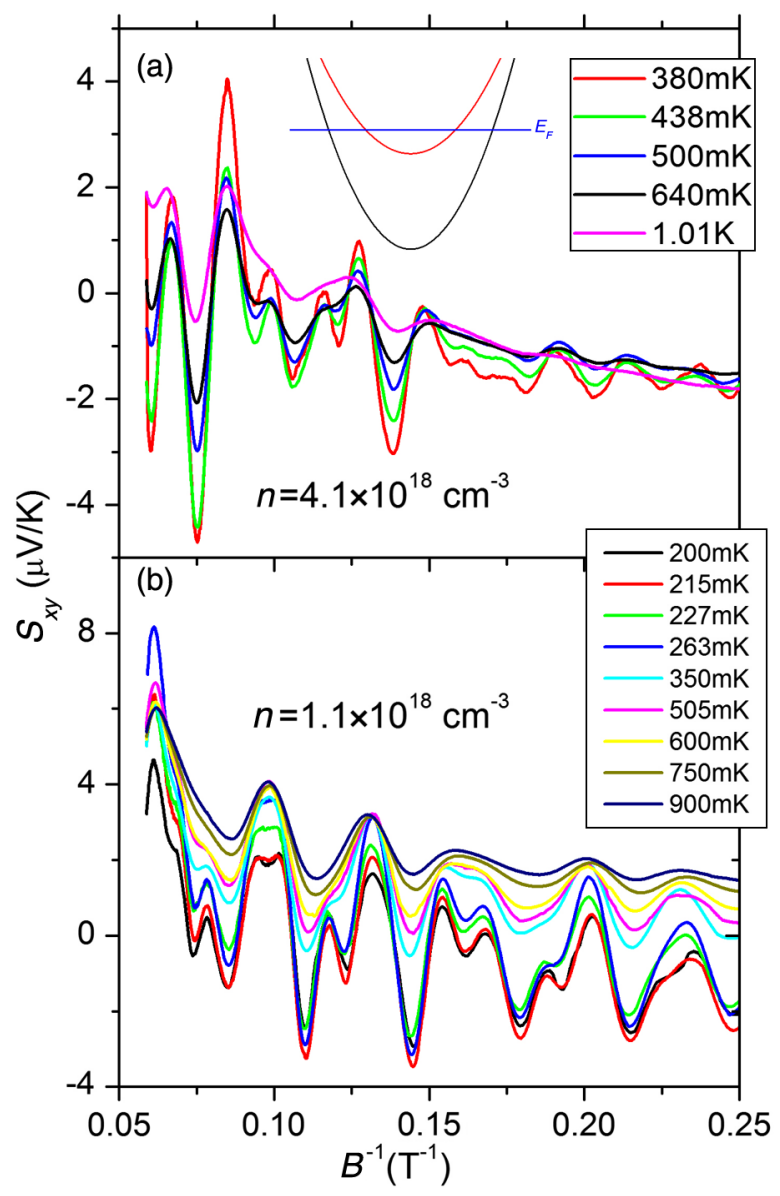

FIG. 2. Nernst signal as a function of the inverse of the magnetic field for two carrier concentrations. The quantum oscillations display a complex structure with multiple periodicities. The inset shows a sketch of the position of Fermi energy relative to the two lowest bands at the $\Gamma$ point. 
magnetic field for seven samples with different carrier concentrations kept at the same temperature $(0.5 \mathrm{~K})$. As is seen in the figure, quantum oscillations are barely detectable in the sample with the highest carrier density. As $n$ decreases, the amplitude of oscillations grows and their frequency shrinks. Giant oscillations of the Nernst effect with the approach of the quantum limit were previously observed in semimetallic bismuth [21] and graphite [22], as well as doped $\mathrm{Bi}_{2} \mathrm{Se}_{3}$ [23]. A property that these three systems share with lightly doped $\mathrm{SrTiO}_{3}$ is that their Fermi surface is an extremely small portion of the Brillouin zone. A $10 \mathrm{~T}$ magnetic field truncates such a tiny Fermi surface into a few Landau tubes. In such a context, each time a squeezed Landau tube leaves the Fermi surface, the Nernst signal peaks. The Nernst quantum oscillations are concomitant with Shubnikov-de Haas oscillations. For the lowest Landau indexes, however, while the oscillating component of resistivity is a small fraction of the overall signal, the oscillating part of the Nernst coefficient dominates the monotonous background [22], making the analysis of the Nernst data straightforward.

At low magnetic fields, the monotonous Nernst signal is expected to be affected by fluctuating superconductivity. In a superconductor, fluctuations of the superconducting order parameter can generate a Nernst signal above $T_{c}$. Theory has quantified the magnitude of the signal due to the Gaussian fluctuations of the superconducting order parameter in both two and three dimensions [27]. On the other hand, the contribution of normal quasiparticles to the Nernst signal is proportional to the ratio of their mobility to their Fermi energy [28]. In a dirty high-density twodimensional superconductor with low electron mobility and large Fermi energy, the quasiparticle contribution is small, and the superconducting Nernst signal is detectable in a large temperature window above $T_{c}$ [29]. In slightly doped bulk $\mathrm{SrTiO}_{3}$, in contrast, the electron mobility is large, the Fermi energy is small, and the Nernst signal is therefore dominated by the quasiparticle contribution. (See the Supplemental Material for a comparison of the orders of magnitude of the quasiparticle and superconducting contributions to the Nernst signal [26]).

As is seen in Fig. 1, in the intermediate doping range, the oscillations display a complex structure, and several frequencies are detectable. For samples with larger carrier densities $\left(n \geq 1.05 \times 10^{18} \mathrm{~cm}^{-3}\right)$, the spectrum of oscillations is indicative of the presence of more than one component of the Fermi surface. For the lowest doping level, the structure becomes remarkably simpler.

Figure 2 displays detailed data at different temperatures for two low-density samples. Quantum oscillations show a complex structure. At $n=4.1 \times 10^{18} \mathrm{~cm}^{-3}$, more than one frequency are clearly detectable, indicating that the Fermi level is above the bottom of the second band and that there are two occupied bands. At $n=1.05 \times 10^{18} \mathrm{~cm}^{-3}$, we detect two sets of peaks with slightly different frequencies (26.8 and 28.3 T). These two almost identical
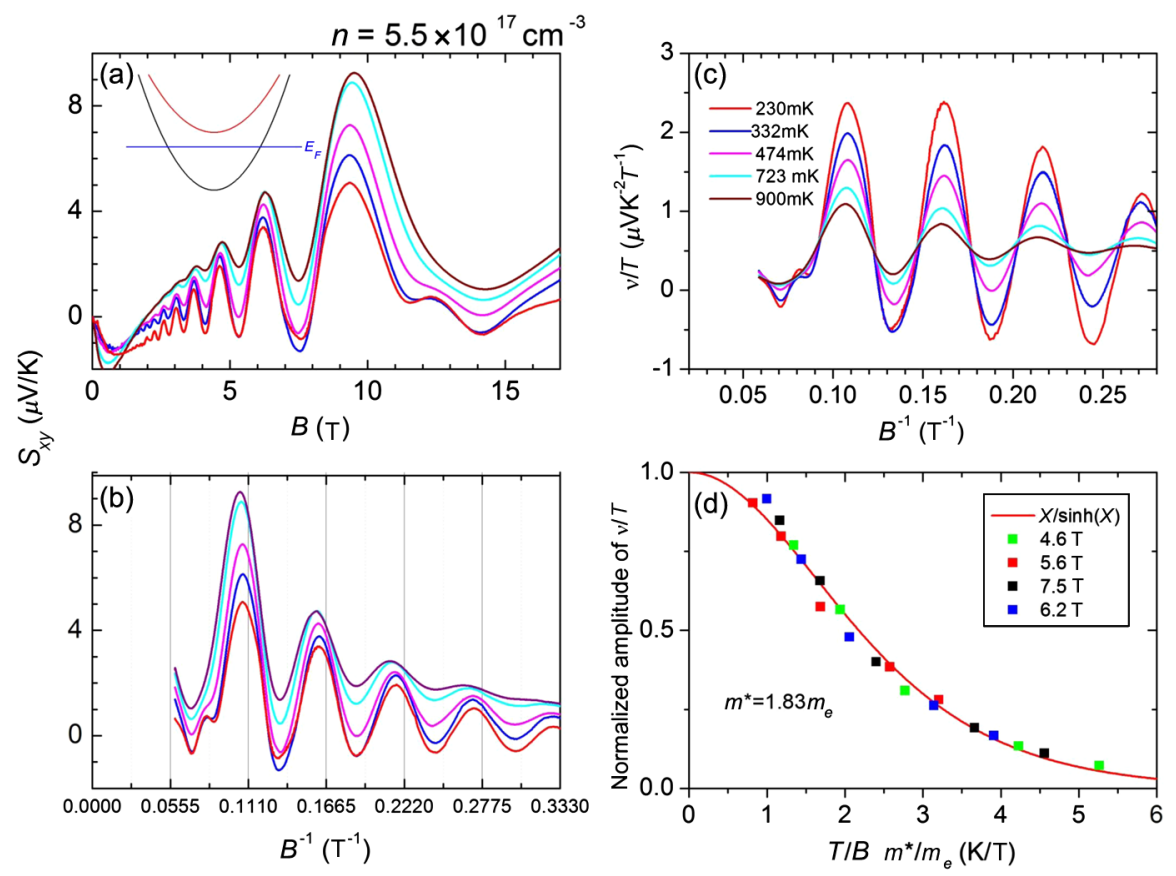

FIG. 3. Nernst quantum oscillations as a function of (a) the magnetic field and (b) the inverse of the magnetic field at different temperatures for the sample with a carrier density of $5.5 \times 10^{17} \mathrm{~cm}^{-3}$. There is a single periodicity, with the lowest peak displaying Zeeman splitting. (c) The variation of the Nernst coefficient $\left(\nu=S_{x y} / B\right.$ ) divided by temperature, with the inverse of the magnetic field at different temperatures. (d) The amplitude of oscillations in $\nu / T$ for different magnetic fields and temperatures. The solid line represents the damping expected in the Lifshitz-Kosevitch theory for an effective mass of $1.83 m_{e}$. 

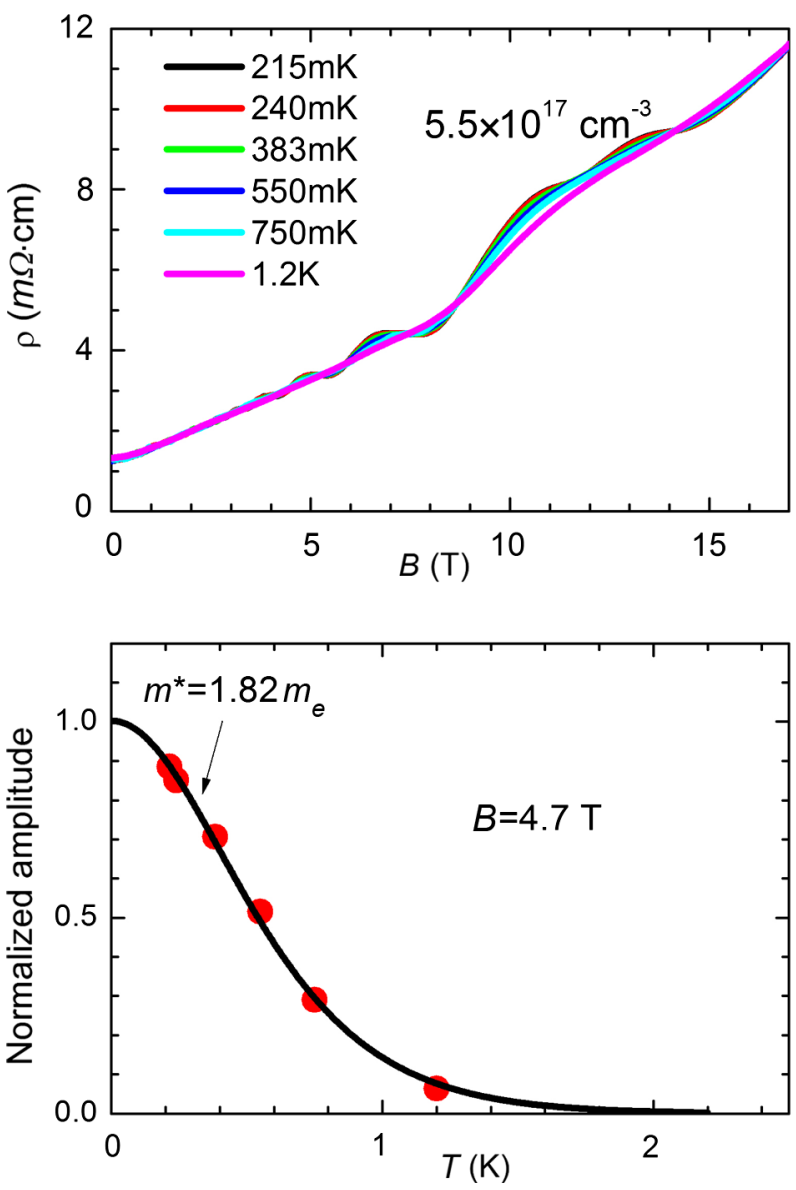

FIG. 4. Top: The Shubnikov-de Haas effect is visible in the magnetoresistance of the lowest doped $\mathrm{SrTiO}_{3}$ sample. Bottom: The effective mass obtained from the resistivity data is close to the one obtained by the analysis of the Nernst data.

frequencies may correspond to two different bands or a single spin-split band.

One major result reported here is the unambiguous detection of single-frequency quantum oscillations at a carrier density of $n=5.5 \times 10^{17} \mathrm{~cm}^{-3}$. The data for this sample are shown in Fig. 3. As is seen in Figs. 3(a) and 3(b), there is only a single periodicity for all temperatures. Moreover, the Nernst peak for the lowest detected Landau level is split at low enough temperatures. The data immediately settle the periodicity $\left(0.0555 T^{-1}\right)$ corresponding to a frequency of $18.2 \mathrm{~T}$. The distance between the two spin-split peaks quantifies the relative magnitudes of the Zeeman and cyclotron energies. Zeeman splitting had not been clearly detected in the system until the present study.

Let us compare these findings with the conclusions of recent ab initio band calculations [30]. As the insulator is doped by $n$-type carriers, the first available band to be filled is a threefold degenerate one associated with a $\mathrm{Ti} 3 d$ orbital. The threefold degeneracy is lifted first by spinorbit coupling and then by the crystal electric field. Filling these bands successively generates three interpenetrating ellipsoids at the center of the Brillouin zone. According to these calculations, at a critical doping of $x_{c 1}=4 \times 10^{-5}$ carriers per formula unit [30], the second band starts to be filled. The corresponding carrier density is $n_{c 1}=$ $6.8 \times 10^{17} \mathrm{~cm}^{-3}$, slightly larger than our most dilute sample. Therefore, there is a fair agreement between experiment and theory.

According to the Onsager relation, the frequency of quantum oscillations $F$ is set by the extremal cross section of the Fermi surface $A_{k}: F=\left(\frac{\hbar}{2 \pi e}\right) A_{k}$. A frequency of $18.2 \mathrm{~T}$ implies a cross section of $A=0.18 \mathrm{~nm}^{-2}$. Now, for a spherical Fermi surface, such a cross section corresponds to $4.6 \times 10^{17} \mathrm{~cm}^{-3}$, very close to what is given by measuring the Hall resistivity $\left(n=\frac{1}{e R_{H}}=\right.$ $\left.5.5 \times 10^{17} \mathrm{~cm}^{-3}\right)$. We conclude that, at this concentration, the Fermi surface is a slightly anisotropic sphere, in agreement with the expectations of the band calculations, which do not find sizeable anisotropy in dispersion along [100], [101], and [110] for $n<n_{c 1}$.

In the Lifshitz-Kosevitch theory, the thermal smearing of quantum oscillations is set by the magnitude of cyclotron mass $m^{*}$. As the temperature increases, the ratio of thermal energy to the cyclotron energy increases. Therefore, the amplitude of oscillations decreases, following $\frac{X}{\sinh X}$ dependence. Here, $X$ is a dimensionless temperature normalized by magnetic field and effective mass: $X=\left(\frac{2 \pi^{2} k_{B}}{\hbar e}\right)\left(\frac{m^{*} T}{B}\right)$. As in the case of $\mathrm{Bi}_{2} \mathrm{Se}_{3}$ [23], we find that employing this formalism to oscillations of the Nernst coefficient divided by temperature $(\nu / T)$ [see Fig. 3(c)] leads to an accurate determination of the cyclotron mass. Figure 3(d) compares the experimentally resolved amplitudes at different fields and temperatures with the expectations of the Lifshitz-Kosevitch equation, assuming $m^{*}=1.83 m_{e}$. Figure 4 displays the resistivity and its quantum oscillations in the same sample. As is seen in the bottom panel of Fig. 4, a fit to our Shubnikov-de Haas data yields a quasi-identical value for the effective mass of $m^{*}=(1.82 \pm 0.05) m_{e}$.

The effective mass obtained here is in fair agreement with what was estimated by measuring the plasma frequency $\left[m^{*}=(2 \pm 0.3) m_{e}\right]$ [31]. Note, however, that the latter measurements are performed at higher concentrations, where several bands with possibly different masses are present. The cyclotron mass obtained here is 2.5 times larger than the band mass $\left(0.7 m_{e}[30,31]\right)$. The mass enhancement is presumably due to a combination of electron-phonon and electron-electron interactions.

The Fermi temperature of this dilute liquid of electrons can be estimated in two distinct ways. First, the magnitude of cyclotron mass together with the size of the Fermi wave vector $k_{F}$ obtained from the frequency of the quantum oscillations can be plugged into

$$
k_{B} T_{F}=\frac{\hbar^{2} k_{F}^{2}}{2 m^{*}}
$$

to obtain a Fermi temperature of $13.5 \mathrm{~K}$. 
Another way to estimate the Fermi temperature is to put the magnitude of the Seebeck coefficient under scrutiny. In a wide variety of correlated metals, the slope of the Seebeck coefficient in the zero-temperature limit is inversely proportional to the Fermi temperature, as is expected in the semiclassical transport theory for a Fermi liquid [32]. In doped $\mathrm{SrTiO}_{3}$, the magnitude of the Seebeck coefficient in the intermediate temperature range is known to be remarkably large [33]. Figures 5(a) and 5(b) display the evolution of the low-temperature Seebeck coefficient in $\mathrm{SrTiO}_{3}$ with doping. As is seen in the figure, as the carrier density lowers, the diffusive component of the Seebeck coefficient increases. In the sample with the lowest carrier concentration $\left(n=5.5 \times 10^{17} \mathrm{~cm}^{-3}\right)$, the magnitude of $S / T$ becomes as large as $-22 \mu \mathrm{V} \mathrm{K}^{-2}$. Assuming an energy-independent mean free path and a spherical Fermi surface, a Fermi temperature of $T_{F}=12.9 \mathrm{~K}$ is obtained using the equation

$$
\left|\frac{S}{T}\right|=\frac{\pi^{2}}{3} \frac{k_{B}}{e} \frac{1}{T_{F}}
$$

Thus, data obtained from two independent probes converge to a Fermi temperature as low as $13 \mathrm{~K}$. The energy gap between valence and conduction bands in $\mathrm{SrTiO}_{3}$ is as large as $3 \mathrm{eV}$. By removing one oxygen atom out of $10^{5}$ (assuming that each oxygen vacancy liberates two potentially mobile electrons), one can create a metal with a chemical potential as small as $1.1 \mathrm{meV}$ on the top of this gap. It is remarkable that this well-defined chemical potential can be achieved in spite of unavoidable inhomogeneities in dopant distribution. Long-range screening, which damps local bandbending effects, appears to be the key factor here. This result is the first major outcome of this investigation.

This dilute liquid of electrons becomes a superconductor below a critical temperature of $T_{c}=86 \mathrm{mK}$. This second unexpected result is illustrated in Fig. 5(c), which shows the resistive transition. The width of transition (defined as the difference between the temperatures of $10 \%$ and $90 \%$ drops in resistivity) is $25 \mathrm{mK}$, to be compared with a width of $30 \mathrm{mK}$ in a sample with a $T_{c}$ of $180 \mathrm{mK}$ and a carrier density of $4.1 \times 10^{18} \mathrm{~cm}^{-3}$
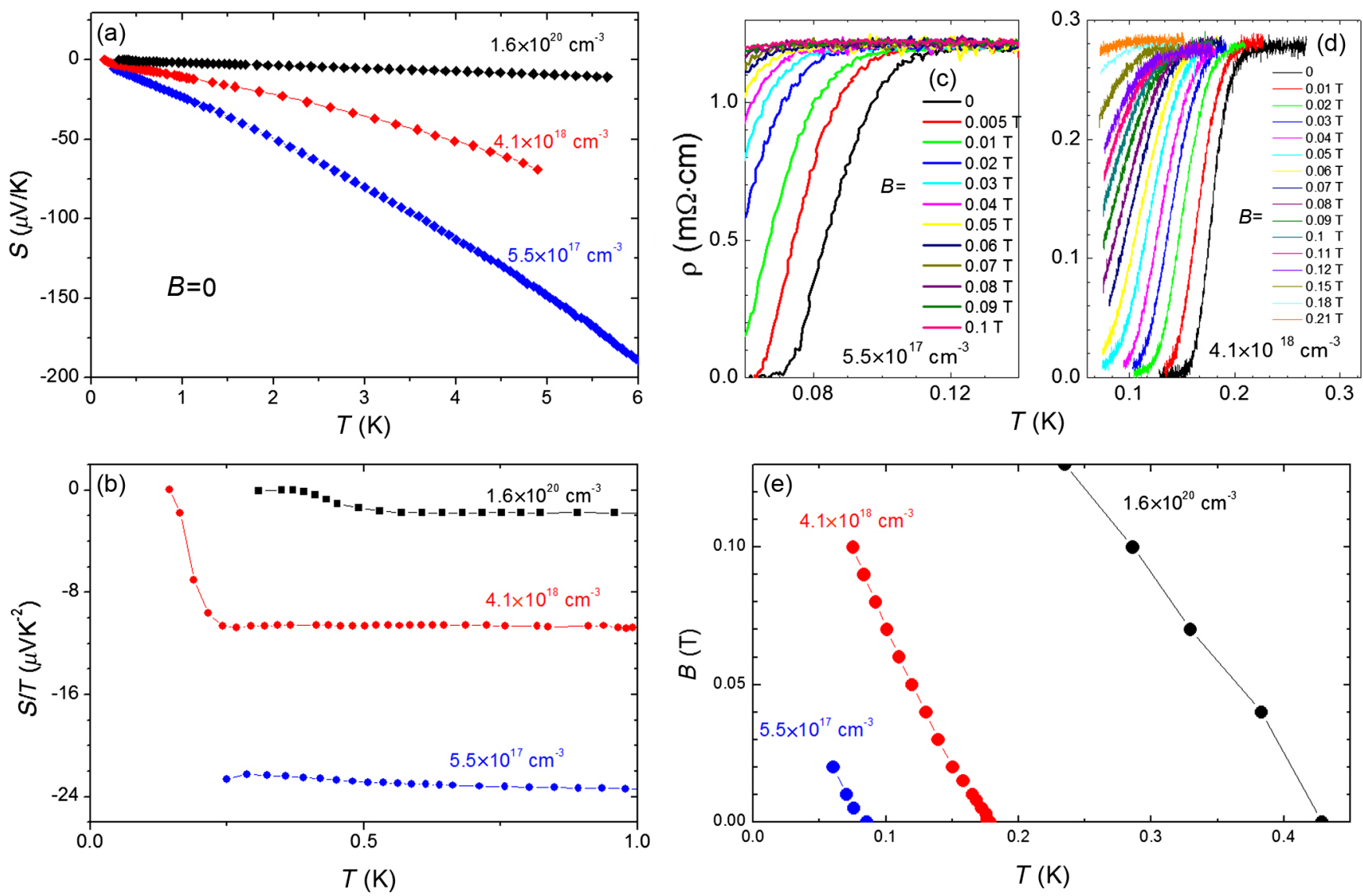

FIG. 5. (a) The temperature dependence of the zero-field Seebeck coefficient for three different carrier concentrations. (b) The temperature dependence of $S / T$. As is seen in the figure, the diffusive component of thermopower becomes larger with underdoping, which is indicative of a decrease in the Fermi energy. (c),(d) Superconducting resistive transition for two different carrier concentrations in the presence of a magnetic field. (e) Temperature dependence of the upper critical field near $T_{c}$ (defined as the temperature at which resistivity drops by half) for three carrier concentrations. 
[Fig. 5(d)]. As is seen in Figs. 5(c)-5(e), the superconducting transition is easily suppressed by the application of a small magnetic field.

Figure 6 details the evolution of superconductingand normal-state properties with doping. As is seen in Fig. 6(a), the evolution of $T_{c}$ with carrier concentration in our samples is comparable with those reported in Ref. [11], save for the persistence of a long tail on the underdoped side of the phase diagram. Figure 6(b) presents the evolution of frequencies resolved by quantum oscillations with carrier doping. In most samples, in addition to the main frequency, others are resolved, pointing to the existence of several quantized orbits of

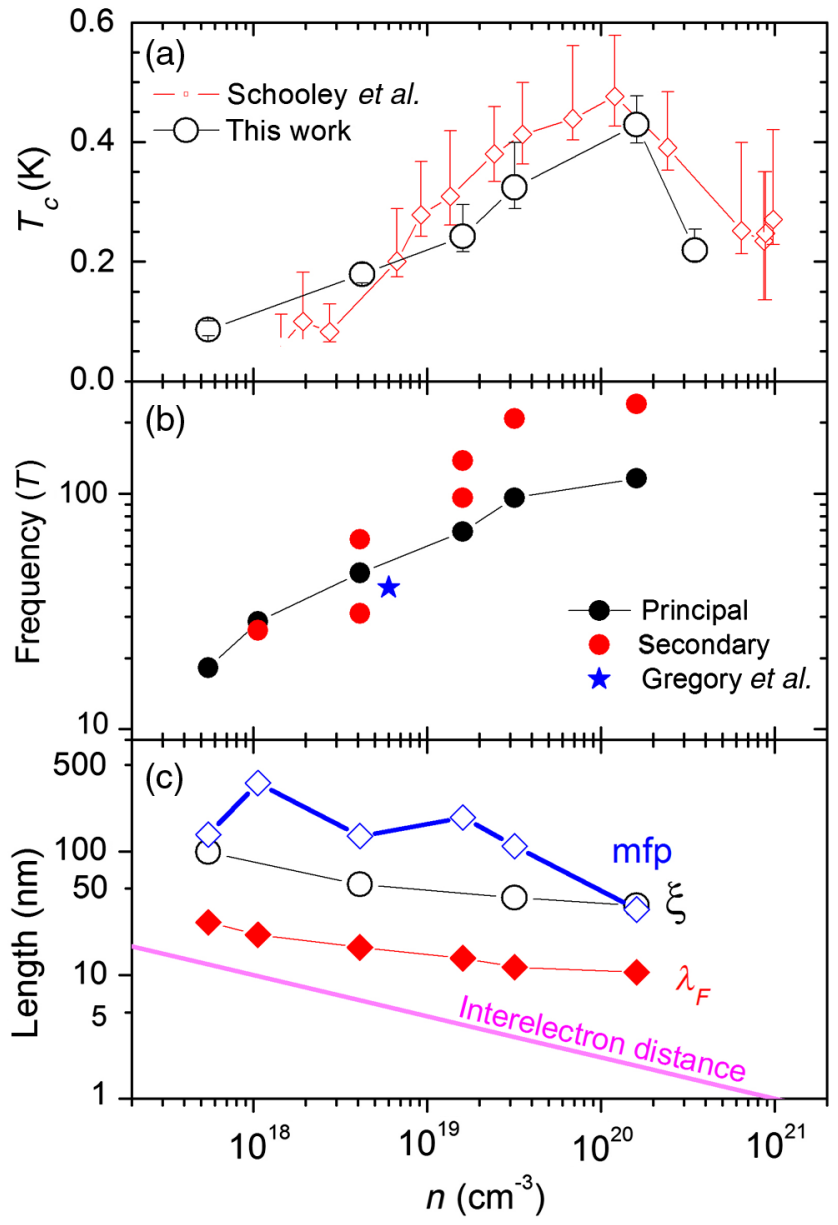

FIG. 6. (a) Variation of the critical temperature with carrier concentration according to this work and Ref. [11]. The width of the superconducting transition in each case is represented by error bars. (b) Evolution of detected frequencies as a function of doping. For carrier concentrations larger than $10^{18} \mathrm{~cm}^{-3}$, the oscillations display more than one frequency. The frequency and carrier density reported in Ref. [16] are also shown. (c) Variation of four relevant length scales, namely, the electronic mean free path (mfp), the average Fermi wavelength $\lambda_{F}$ of the electrons of the main band, the superconducting coherence length $\xi$, and the interelectron distance. electrons as successive bands are filled. For comparison, Fig. 6(b) shows the unique frequency previously reported in the bulk [16]. Finally, Fig. 6(c) compares the evolution of four different length scales of the system: The average interelectron distance (estimated from carrier density, $d_{e e}=n^{-1 / 3}$ ); the average Fermi wavelength (calculated from the Fermi wave vector $k_{F}$ of the main band obtained from the principal frequency, $\lambda_{F}=\frac{2 \pi}{k_{F}}$ ); the mean free path (estimated from the measured Hall mobility $\mu_{H}$ and $\left.k_{F}, \ell_{e}=\frac{\hbar k_{F}}{e} \mu_{H}\right)$; and finally the superconducting coherence length (derived from the slope of the upper critical field, $\xi^{-2}=\left.\frac{2 \pi}{\Phi_{0}} 0.69 T_{c} \frac{d H_{c 2}}{d T}\right|_{T_{c}}$ ).

Comparing these length scales, we can make several observations. With a static dielectric constant as large as $2.5 \times 10^{4}$ [9] and an effective mass of $1.8 m_{e}$, the Bohr radius is of the order of $0.7 \mu \mathrm{m}$. Therefore, the Mott criterion for metallicity $\left(a_{B}^{*} n^{1 / 3} \gg 0.26\right)$ [8] is easily satisfied. Since electrons can travel much further than the interdopant distance, the superconductor remains in the clean limit $\left(\ell_{e}>\xi\right)$ in our range of study. Finally, the system avoids localization because $\ell_{e} \gg$ $\lambda_{F}$. As is noted previously [30], this superconductor stays in the BCS side of the Bose-Einstein condensate BCS crossover. Even for the lowest concentration, the average size of Cooper pairs $(\xi \simeq 100 \mathrm{~nm})$ is much longer than the interelectron distance $\left(d_{e e}=12 \mathrm{~nm}\right)$.

Superconductivity at such a low carrier density has never been explored in two-dimensional $\mathrm{SrTiO}_{3}$ because the electron mean free path is much shorter in the latter system. A three-dimensional carrier density of $5.5 \times 10^{17} \mathrm{~cm}^{-3}$ corresponds to a $2 \mathrm{D}$ carrier density as low as $n_{2 \mathrm{D}}=6.7 \times 10^{11} \mathrm{~cm}^{-2}$. Before attaining such a low carrier density, the superconducting ground state is destroyed by a superconductor-to-insulator transition [34], which occurs when $k_{F} \ell \sim 1$.

\section{CONCLUSION}

Bulk $\mathrm{SrTiO}_{3}$ with an oxygen deficiency of $10^{-5}$ per formula unit is a superconductor. This carrier density is 3-4 orders of magnitude lower than what has been reported in conventional semiconducting superconductors. Indeed, boron-doped silicon, diamond, or silicon carbide all require a few-percent doping to display superconductivity [35]. In contrast to $\mathrm{SrTiO}_{3}$, the mobility in the latter systems is too low for quantum oscillations, and a well-defined Fermi surface has not been observed in the normal state.

$\mathrm{SrTiO}_{3}$ is distinct by its single-component, extremely small, and barely anisotropic Fermi surface. Such a simple Fermi-surface topology is rare among superconductors. It is a well-known fact that noble and alkali metals stand out in the periodical table by their refusal to superconduct. More importantly, the extreme low density generates several peculiar features. The Fermi velocity $\left(v_{F}=\frac{\hbar k_{F}}{m^{*}}\right)$ 
becomes as low as $15 \mathrm{~km} \mathrm{~s}^{-1}$, which is almost as slow as sound velocity. The Fermi temperature is an order of magnitude lower than the Debye temperature, a situation that is never met in a conventional superconductor but that is common in a heavy-fermion superconductor [36]. The inversion of Debye and Fermi energy scales has profound consequences for the relative weight of Coulomb repulsion and phonon-mediated attraction between electrons [37] and is a serious and perhaps insurmountable challenge for any theory of superconductivity based on phononelectron interaction.

The $T_{c} / T_{F}$ ratio in dilute $\mathrm{SrTiO}_{3}$ is somewhat lower than what is reported in optimally doped unconventional (cuprate, heavy-fermion, and iron-based) superconductors. But, it is an order of magnitude larger than in elemental conventional superconductors and even somewhat larger than in $\mathrm{Sr}_{2} \mathrm{RuO}_{4}$, a correlated metal believed to be a spintriplet superconductor [38]. In the latter case, $T_{c}=1.5 \mathrm{~K}$ and each of the three bands has a Fermi temperature of the order of $T_{F} \sim 1000 \mathrm{~K}$.

The microscopic mechanism of pair formation in bulk $\mathrm{SrTiO}_{3}$ remains a wide-open question. Our current knowledge of the Fermi surface, a single valley at the center of the Brillouin zone, leaves no room for intervalley phonons invoked by the oldest theoretical attempt to explain the existence of a superconducting dome in this system [12]. On the other hand, the scenario invoking the exchange of plasmons between electrons [39] appears to be reconcilable with the persistence of superconductivity in a dilute one-band context. Little is known about the superconducting order parameter other than the reported observation of a multigap superconductivity in the doping range above $10^{19} \mathrm{~cm}^{-3}$ [13]. Future experiments that employ bulk probes such as thermal conductivity or penetration depth are planned to probe the superconducting gap and to find nodes, if they exist.

A detailed analysis of quantum oscillations would open a new window to the problem. Since an accurate determination of the Fermi-surface topology and effective mass would lead to the density of states at arbitrary doping, and since the BCS formula $\left(T_{c}=\Theta \exp \left[\frac{-1}{N(0) V}\right]\right)$ links $T_{c}$ with an energy scale $\Theta$, an interaction parameter $V$, and the density of states $N(0)$, competing scenarios can be tested by comparing their expectations for the variations of $V$ and $\Theta$.

\section{ACKNOWLEDGMENTS}

We acknowledge stimulating discussions with Harold Hwang, Dirk van der Marel, Kazuamasa Miyake, and Yasutami Tanaka and are grateful to Young Jun Chang for providing us a number of $\mathrm{Nb}$-doped $\mathrm{SrTiO}_{3}$ samples. This work is supported by Agence Nationale de la Recherche as part of the QUANTHERM and SUPERFIELD projects. X.L. is supported by the China Research Council.
[1] J.F. Schooley, W.R. Hosler, and M.L. Cohen, Superconductivity in Semiconducting $\mathrm{SrTiO}_{3}$, Phys. Rev. Lett. 12, 474 (1964).

[2] J. K. Hulm, M. Ashkin, D. W. Deis, and C. K. Jones, Superconductivity in Semiconductors and Semimetals, Prog. Low Temp. Phys. 6, 205 (1970).

[3] X. Blase, E. Bustarret, C. Chapelier, T. Klein, and C. Marcenat, Superconducting Group-IV Semiconductors, Nat. Mater. 8, 375 (2009).

[4] A. Ohtomo and H. Y. Hwang, A High-Mobility Electron Gas at the $\mathrm{LaAlO}_{3} / \mathrm{SrTiO}_{3}$ Heterointerface, Nature (London) 427, 423 (2004).

[5] A.F. Santander-Syro et al., Two-Dimensional Electron Gas with Universal Subbands at the Surface of $\mathrm{SrTiO}_{3}$, Nature (London) 469, 189 (2011).

[6] N. Reyren et al., Superconducting Interfaces between Insulating Oxides, Science 317, 1196 (2007).

[7] J. Biscaras, N. Bergeal, A. Kushwaha, T. Wolf, A. Rastogi, R.C. Budhani, and J. Lesueur, Two-Dimensional Superconductivity at a Mott Insulator/Band Insulator Interface $\mathrm{LaTiO}_{3} / \mathrm{SrTiO}_{3}$, Nat. Commun. 1, 89 (2010).

[8] P. P. Edwards and M. J. Sienko, Universality Aspects of the Metal-Nonmetal Transition in Condensed Media, Phys. Rev. B 17, 2575 (1978).

[9] K. A. Müller and H. Burkard, $\mathrm{SrTiO}_{3}$ : An Intrinsic Quantum Paraelectric below 4 K, Phys. Rev. B 19, 3593 (1979).

[10] A. Spinelli, M. A. Torija, C. Liu, C. Jan, and C. Leighton, Electronic Transport in Doped $\mathrm{SrTiO}_{3}$ : Conduction Mechanisms and Potential Applications, Phys. Rev. B 81, 155110 (2010).

[11] J. F. Schooley, W. R. Hosler, E. Ambler, J.H. Becker, M.L. Cohen, and C.S. Koonce, Dependence of the Superconducting Transition Temperature on Carrier Concentration in Semiconducting $\mathrm{SrTiO}_{3}$, Phys. Rev. Lett. 14, 305 (1965).

[12] C. S. Koonce, M. L. Cohen, J. F. Schooley, W. R. Hosler, and E.R. Pfeiffer, Superconducting Transition Temperatures of Semiconducting $\mathrm{SrTiO}_{3}$, Phys. Rev. 163, 380 (1967).

[13] G. Binnig, A. Baratoff, H.E. Hoenig, and J. G. Bednorz, Two-Band Superconductivity in Nb-Doped $\mathrm{SrTiO}_{3}$, Phys. Rev. Lett. 45, 1352 (1980).

[14] H. Suzuki, H. Bando, Y. Ootuka, I. H. Inoue, T. Yamamoto, K. Takahashi, and Y. Nishihara, Superconductivity in Single-Crystalline $\mathrm{Sr}_{1-x} \mathrm{La}_{x} \mathrm{TiO}_{3}$, J. Phys. Soc. Jpn. 65, 1529 (1996).

[15] H.P.R. Frederikse, W.R. Hosler, W.R. Thurber, J. Babiskin, and P.G. Siebenmann, Shubnikov-de Haas Effect in $\mathrm{SrTiO}_{3}$, Phys. Rev. 158, 775 (1967).

[16] B. Gregory, J. Arthur, and G. Seidel, Measurements of the Fermi Surface of $\mathrm{SrTiO}_{3}$ : Nb, Phys. Rev. B 19, 1039 (1979).

[17] Y. Kozuka, M. Kim, C. Bell, B. G. Kim, Y. Hikita, and H. Y. Hwang, Two-Dimensional Normal-State Quantum Oscillations in a Superconducting Heterostructure, Nature (London) 462, 487 (2009).

[18] M. Ben Shalom, A. Ron, A. Palevski, and Y. Dagan, Shubnikov-de Haas Oscillations in $\mathrm{SrTiO}_{3} / \mathrm{LaAlO}_{3}$ Interface, Phys. Rev. Lett. 105, 206401 (2010). 
[19] A. D. Caviglia, S. Gariglio, C. Cancellieri, B. Sacépé, A. Fête, N. Reyren, M. Gabay, A. F. Morpurgo, and J.-M. Triscone, Two-Dimensional Quantum Oscillations of the Conductance at $\mathrm{LaAlO}_{3} / \mathrm{SrTiO}_{3}$ Interfaces, Phys. Rev. Lett. 105, 236802 (2010).

[20] M. Kim, C. Bell, Y. Kozuka, M. Kurita, Y. Hikita, and H. Y. Hwang, Fermi Surface and Superconductivity in Low-Density High-Mobility $\delta$-doped $\mathrm{SrTiO}_{3}$, Phys. Rev. Lett. 107, 106801 (2011).

[21] K. Behnia, M-.A. Méasson, and Y. Kopelevich, Oscillating Nernst-Ettingshausen Effect in Bismuth across the Quantum Limit, Phys. Rev. Lett. 98, 166602 (2007).

[22] Z. Zhu, H. Yang, B. Fauqué, Y. Kopelevich, and K. Behnia, Nernst Effect and Dimensionality in the Quantum Limit, Nat. Phys. 6, 26 (2009).

[23] B. Fauqué, N.P. Butch, P. Syers, J. Paglione, S. Wiedmann, A. Collaudin, B. Grena, U. Zeitler, and K. Behnia, Magnetothermoelectric Properties of $\mathrm{Bi}_{2} \mathrm{Se}_{3}$, Phys. Rev. B 87, 035133 (2013).

[24] H. P. R. Frederikse, W. R. Hosler, and W. R. Thurber, Magnetoresistance of Semiconducting $\mathrm{SrTiO}_{3}$, Phys. Rev. 143, 648 (1966).

[25] H. P. R. Frederikse and W. R. Hosler, Hall Mobility in $\mathrm{SrTiO}_{3}$, Phys. Rev. 161, 822 (1967).

[26] See Supplemental Material at http://link.aps.org/ supplemental/10.1103/PhysRevX.3.021002 for details on sample characterization and a brief discussion of different sources of a Nernst signal.

[27] I. Ussishkin, S. L. Sondhi, and D. A. Huse, Gaussian Superconducting Fluctuations, Thermal Transport, and the Nernst Effect, Phys. Rev. Lett. 89, 287001 (2002).

[28] K. Behnia, The Nernst Effect and the Boundaries of the Fermi Liquid Picture, J. Phys. Condens. Matter 21, 113101 (2009).

[29] A. Pourret, H. Aubin, J. Lesueur, C. A. Marrache-Kikuchi, L. Bergé, L. Dumoulin, and K. Behnia, Observation of the Nernst Signal Generated by Fluctuating Cooper Pairs, Nat. Phys. 2, 683 (2006).
[30] D. van der Marel, J. L. M. van Mechelen, and I. I. Mazin, Common Fermi-Liquid Origin of $T^{2}$ Resistivity and Superconductivity in n-type $\mathrm{SrTiO}_{3}$, Phys. Rev. B 84, 205111 (2011).

[31] J.L. M. van Mechelen, D. van der Marel, C. Grimaldi, A. B. Kuzmenko, N.P. Armitage, N. Reyren, H. Hagemann, and I. I. Mazin, Electron-Phonon Interaction and Charge Carrier Mass Enhancement in $\mathrm{SrTiO}_{3}$, Phys. Rev. Lett. 100, 226403 (2008).

[32] K. Behnia, D. Jaccard, and J. Flouquet, On the Thermoelectricity of Correlated Electrons in the ZeroTemperature Limit, J. Phys. Condens. Matter 16, 5187 (2004).

[33] T. Okuda, K. Nakanishi, S. Miyasaka, and Y. Tokura, Large Thermoelectric Response of Metallic Perovskites: $\mathrm{Sr}_{1-x} \mathrm{La}_{x} \mathrm{TiO}_{3}(0<\sim x<\sim 0.1)$, Phys. Rev. B 63, 113104 (2001).

[34] A. D. Caviglia, S. Gariglio, N. Reyren, D. Jaccard, T. Schneider, M. Gabay, S. Thiel, G. Hammerl, J. Mannhart, and J.-M. Triscone, Electric Field Control of the $\mathrm{LaAlO}_{3} / \mathrm{SrTiO}_{3}$ Interface Ground State, Nature (London) 456, 624 (2008).

[35] M. Kriener, Y. Maeno, T. Oguchi, Z.-A. Ren, J. Kato, T. Muranaka, and J. Akimitsu, Specific Heat and Electronic States of Superconducting Boron-Doped Silicon Carbide, Phys. Rev. B 78, 024517 (2008).

[36] Z. Fisk, H.R. Ott, T.M. Rice, and J.L. Smith, Heavy-Electron Metals, Nature (London) 320, 124 (1986).

[37] P. G. de Gennes, Superconductivity of Metals and Alloys (Benjamin, New York, 1966).

[38] A.P. Mackenzie and Y. Maeno, The Superconductivity of $\mathrm{Sr}_{2} \mathrm{RuO}_{4}$ and the Physics of Spin-Triplet Pairing, Rev. Mod. Phys. 75, 657 (2003).

[39] Y. Takada, Theory of Superconductivity in Polar Semiconductors and Its Application to N-Type Semiconducting $\mathrm{SrTiO}_{3}$, J. Phys. Soc. Jpn. 49, 1267 (1980). 